\title{
Presentación Defensa de la competencia en España: un análisis sectorial
}

\author{
Juan Luis Jiménez González \\ Universidad de Las Palmas de Gran Canaria
}

La política de defensa de la competencia es uno de los ámbitos de intervención del Estado en las economías modernas que tiene un mayor impacto microeconómico sobre el bienestar social. Frenar la existencia de prácticas abusivas, colusorias, comportamientos predatorios, etcétera, modifica la realidad de cualquier mercado y afecta a los agentes (consumidores, empresas y el propio Estado) que participan en él. Pero, en muchas ocasiones, se olvidan las repercusiones que tiene la defensa de la competencia también a nivel macroeconómico y que son igualmente relevantes.

Es un resultado ampliamente contrastado que la competencia constituye uno de los pilares básicos del crecimiento económico y la eficiencia, así como un elemento para el control de la inflación. Transformar el benchmark utópico de la competencia perfecta en realidad debería ser uno de los objetivos básicos de cualquier gobierno preocupado por maximizar el bienestar social.

En las últimas décadas hemos asistido a cambios sustanciales en muchos mercados en España: liberalizaciones sectoriales, eliminación de antiguos monopolios, procesos de concentración empresarial, cambios en los patrones de comportamiento de los consumidores, modificaciones en la Ley de Competencia, y, finalmente, un proceso de agregación de las tareas de supervisión y regulación en un único organismo: la Comisión Nacional de los Mercados y la Competencia (CNMC), creada en 2013. Todos estos cambios han supuesto modificaciones de calado en la mayoría de los sectores económicos, a los que los agentes han ido adaptándose y modificando sus comportamientos.

Al evaluar todos estos cambios (normativos, institucionales, decisiones de empresas y consumidores), surgen varias cuestiones relativas a la situación de la competencia en España, y su comparación con la realidad de otros países de nuestro entorno: ¿tenemos una política de defensa de la competencia adecuada para el nivel de desarrollo del país? ¿Disponen las autoridades de competencia de los instrumentos necesarios para alcanzar los objetivos? ¿Se toman decisiones que realmente mejoran el bienestar social en los mercados en los que se intervienen? ¿Se han conseguido mejoras efectivas?

El presente monográfico de Cuadernos Económicos de ICE aborda algunas de estas cuestiones relativas a la evaluación económica de la situación de la competencia y su defensa (a partir de los instrumentos disponibles por las Administraciones Públicas para ello), desde una perspectiva microeconómica, examinando los impactos de esta 
política en diversos sectores en España. La principal motivación de este número es realizar un análisis riguroso del grado de competencia alcanzado en sectores económicos que, en muchos casos, son considerados como estratégicos, tanto por su importancia per se como por sus efectos de arrastre.

Se aporta así una amplia panorámica de sectores relevantes para la economía española, que permite también reflejar qué investigación académica se realiza actualmente en España sobre la política de competencia, así como señalar posibles líneas de investigación futuras en el área. Por la limitación de la extensión del monográfico, hemos dejado fuera algunos otros sectores interesantes desde el punto de vista de la competencia, así como también la evaluación de los impactos macroeconómicos de la política de defensa de la competencia.

Comienza el presente monográfico con un análisis del marco institucional en España. El trabajo de Joan-Ramon Borrell, Juan Luis Jiménez González y Carmen García se centra en un aspecto fundamental: la estabilidad institucional, como base para el correcto desarrollo de cualquier política, en este caso aplicado a la política de competencia. Los autores se aproximan a la efectividad de dicha política por medio del uso de indicadores subjetivos de la misma, utilizados ampliamente en la literatura académica como proxies del marco competitivo que existe en un país.

Utilizando una base de datos de más de 50 países a lo largo de las últimas dos décadas (1995-2014), en el trabajo se describe la alta variabilidad de dicha medida de efectividad percibida en España, además de cómo la amplitud de los ciclos de auge y declive de la política de competencia en España son una anormalidad estadística, ya que son más amplios que los que corresponderían al país por su nivel de desarrollo económico y social. En las conclusiones de este artículo se esboza un problema de calado: la necesidad de una mayor estabilidad e independencia del marco institucional como un factor necesario para una correcta implementación de la política de competencia en España.

Analizado el marco institucional, los tres siguientes trabajos se centran en el análisis de la competencia en el transporte, particularmente en actividades donde las infraestructuras desempeñan un papel relevante: ferrocarriles, puertos y aeropuertos. El artículo de Javier Campos se centra en el primero de estos tres sectores. Tras realizar un repaso a los diferentes modelos de reestructuración ferroviaria y la situación actual de este modo de transporte en Europa, describe el proceso de apertura a la competencia en España, primero en mercancías y, actualmente, en el caso de los pasajeros. Sus conclusiones no son optimistas y sugieren un éxito muy dudoso del proceso de liberalización iniciado. La mejora de los resultados del ferrocarril requiere un compromiso mayor del Gobierno para permitir que la entrada de nuevas empresas en el sector genere verdaderamente una competencia efectiva.

Lourdes Trujillo, Ancor Suárez-Alemán y María Cabrera realizan un desglose de los factores que afectan a la competencia en (y entre) los puertos, con aplicación expresa al sistema de puertos españoles. Para ello los autores describen las principales fuentes endógenas y exógenas de competencia existentes, a partir de las que configuran unos criterios ad hoc para la valoración del grado de competencia 
potencial. Aplicando dichos criterios a los principales puertos españoles, apuntan a una serie de infraestructuras portuarias con niveles bajos de competencia en buena parte de los criterios considerados. En sus recomendaciones de política abogan por revisar los mecanismos de concesión para estas infraestructuras.

El tercero de los trabajos relacionados con el ámbito de las infraestructuras es el de Xavier Fageda. En este caso el autor enfoca su análisis no directamente a la gestión aeroportuaria, sino sobre los efectos que la fusión Iberia-Vueling-Clickair -relevante por la cuota de mercado de estas compañías- tuvo sobre una variable relevante en el sector aeronáutico: las frecuencias aéreas. Su aproximación econométrica a través del estimador en diferencias concluye que la fusión redujo las posibilidades de los consumidores en términos de dicha variable, lo que puede venir explicado por un comportamiento final ciertamente más colusivo. El autor asimismo señala que esta reducción en la competencia entre las firmas puede ser aún más grave si el monopolio que gestiona AENA es finalmente privatizado (parcialmente), instando a las autoridades de la competencia a «vigilar» dicho sector.

Los tres siguientes trabajos estudian lo que, antes de la creación de la CNMC, eran sectores con organismos reguladores propios: electricidad, hidrocarburos y telecomunicaciones. En primer lugar, el trabajo de Aitor Ciarreta y Cristina Pizarro-Irizar desglosa de manera muy ilustrativa el funcionamiento del mercado eléctrico español. Los autores persiguen alcanzar resultados, aunque sean únicamente preliminares, en la evaluación de la reforma del sector eléctrico español llevada a cabo a través de las Leyes de 2013 y 2014, así como diversos Reales Decretos.

Tras describir minuciosamente el marco regulatorio y los principales resultados en la etapa previa a dicha reforma (Subastas CESUR, mercado de renovables, déficit de tarifa), se analiza cómo el cambio del marco regulatorio afecta a los consumidores finales, a las energías renovables, a la cogeneración y al cálculo del déficit tarifario. A partir de los resultados obtenidos, los autores señalan que se ha producido un aumento de los precios finales a los consumidores, un sostenimiento de las energías renovables y un empeoramiento del déficit tarifario. Por tanto, aunque solo de manera tentativa por el poco tiempo que aún ha transcurrido desde su entrada en vigor, un primer balance no parece indicar que se hayan alcanzado resultados más competitivos que en la etapa previa, lo que pondría en duda el éxito de la reforma.

Otro sector con amplia relevancia económica en nuestro país es el de los hidrocarburos. Valeria Bernardo, Juan Luis Jiménez y Jordi Perdiguero se acercan a este sector desde tres perspectivas entrelazadas. En primer término detallan los múltiples casos de prácticas restrictivas de la competencia detectados desde su liberalización; en segundo lugar, se describen las principales normas establecidas por la Administración con el objetivo de introducir competencia en el sector. Por último, y dado que todos los estudios realizados tras la liberalización concluyen que existe un bajo nivel de competencia, los autores se centran en evaluar econométricamente el impacto de una de las últimas medidas implementadas: promover el establecimiento de los operadores minoristas denominados «low cost». Para ello, se estima una ecuación de precios con efectos geográficos incorporados, utilizando datos del área me- 
tropolitana de Barcelona (estaciones de servicio y su geolocalización). Los resultados obtenidos muestran un efecto de reducción en precios como consecuencia de la entrada de estos nuevos operadores, aunque con matices a la hora de valorar el éxito de la medida.

Joan Calzada y Fernando Martínez-Santos elaboran un artículo enfocado al análisis del sector de telecomunicaciones, y en particular desgranan la situación actual de la competencia en el mercado de banda ancha en España. Tras describir la evolución sectorial en el último lustro, los autores comparan el mercado español con el europeo y detallan las cuotas de mercado, estrategias de venta y estructura tarifaria de las empresas que compiten en el mismo. De su análisis descriptivo se concluye el papel positivo que sobre la competencia han tenido los operadores móviles virtuales, que son los que han permitido alcanzar reducciones de precios en los últimos años, si bien señalan que los procesos de concentración empresarial pueden mermar las mejoras alcanzadas.

Los dos últimos trabajos del monográfico abordan temas vinculados al sector de la distribución, un ámbito con varios casos de prácticas restrictivas de la competencia. El artículo realizado por Javier Asensio versa sobre la evolución en la concentración en la distribución de alimentos en España. El enfoque utilizado aborda tanto la descripción de la relación distribuidores-proveedores (habitualmente denominada 'aguas arriba'), como la distribución minorista (ventas a consumidores finales), aportando datos relevantes sobre ambas. Tras esta descripción, el autor analiza econométricamente, utilizando una versión sencilla del modelo de entrada de Bresnahan y Reiss, la relación entre las restricciones comerciales por comunidades autónomas y la oferta comercial por categorías de establecimiento, con el objetivo de evaluar si los cambios normativos liberalizadores que afectan al sector tendrán incidencia en la composición de la oferta minorista. De sus resultados se desprende que cabe esperar un notable impacto en la entrada de establecimientos de tamaño mediano y grande, en mayor volumen a la reducción de los de menor tamaño.

El trabajo de José Manuel Ordóñez de Haro y José Luis Torres evalúa el impacto de la intervención de la Comisión Nacional de Competencia en 2007 sobre la industria del pan en España. Tras describir los detalles de las medidas adoptadas, algunos apuntes básicos sobre el sector, y analizar varios casos concretos en este mercado, los autores utilizan el enfoque de series temporales interrumpidas para cuantificar los efectos de la intervención de la autoridad de defensa de la competencia sobre el precio del pan. De las estimaciones se desprende cómo la recomendación colectiva de subidas de precios detectada en el sector tuvo efecto y, tras la apertura del procedimiento sancionador, el precio del pan se ha mantenido constante, a pesar de las variaciones en el precio de la materia prima.

Resumidos todos los trabajos que conforman este número de Cuadernos Económicos, no quisiera finalizar sin agradecer, en primer lugar, a todos los autores que han participado en este monográfico, por recibir con entusiasmo la invitación a contribuir con sus trabajos y comprometerse con ella, máxime teniendo en cuenta 
los costes de oportunidad en los que se incurre con este tipo de colaboraciones. En segundo lugar, también damos las gracias de forma colectiva a todos los evaluadores que han revisado los artículos y han contribuido con sus comentarios a mejorarlos.

Finalmente, quiero expresar mi agradecimiento al editor Gustavo Nombela, por confiarme la coordinación de este número; y a María Jesús Liceranzu, por su apoyo técnico. Y no quisiera olvidar en mi lista de agradecimientos a todas aquellas empresas, asociaciones, lobbies y también Administraciones Públicas que, o bien tratan de evitar la competencia a toda costa y la convierten en su mayor enemiga, o bien simplemente ignoran sus efectos positivos sobre el conjunto de la sociedad y no apuestan realmente por promoverla: de todos ellos aprendemos y contra ellos seguiremos trabajando. 